\title{
Adjustment of PID control parameters
}

\author{
DAVID DI RUSCIO†
}

Keywords: PID control parameters, automatic tuning.

Methods are presented for the adjustment of PID control parameters, so that the loop transfer function, the sensitivity transfer function or the complementary sensitivity transfer function is tuned to a prescribed amplitude and phase.

\section{Introduction}

Many good methods for the on-line identification of the process parameters needed for tuning PID controllers are available.

A method for the adaption of PID controller parameters in closed loop systems has been presented in Balchen et al.(1989). The method is based on an algorithm where the phase angle $\angle N(j \omega)$ is prescribed, and the corresponding frequency and amplitude of the sensitivity transfer function $N(j \omega)$ are estimated. The proportional gain is adjusted by an integral controller with setpoint equal to a prescribed amplitude of $N$. The integral and derivative times are adjusted proportionally to the inverse of the frequency $\omega$.

A different scheme has been presented in Schei (1991), where the amplitude of the complementary sensitivity transfer function $M(j \omega)$ and the frequency of oscillation are estimated from a describing function approach. The phase angle is fixed to $\angle M(j \omega)=-90^{\circ}$. The controller parameters are updated at each oscillation period in such a way that the amplitude of the loop transfer function $h_{0}(j \omega)$ attains a specified value. The integral and derivative times are adjusted in the same way as in Balchen $e t$ al. (1989).

However, the methods for the adjustment of the proportional gain in these works may be improved.

This note is concerned with the problem of adjusting the PID controller parameters in such a way that some process performance specifications are reached. Such specifications may be prescribed values on the amplitude and phase of the loop, sensitivity or the complementary sensitivity transfer function.

The paper is organized as follows: Section 2 presents the main results. Two examples are presented in Section 3 and some concluding remarks follow in Section 4.

\section{Main results}

The main results in this section are stated in Section 2.1, 2.2 and 2.3. Section 2.1 deals with the loop transfer function $h_{0}(j \omega)$, Section 2.2 considers the sensitivity transfer function $N(j \omega)$ and Section 2.3 is concerned with the complementary

Received 21 January 1992.

$\dagger$ Division of Engineering Cybernetics, Norwegian Institute of Technology, N-7034 Trondheim, Norway. 
sensitivity transfer function $M(j \omega)$ for the adjustment of PID controller parameters. All methods are based on the idea that the integral time $T_{i}$, and the derivative time $T_{d}$ are functions of the frequency, i.e. $T_{i}=T_{i}(\omega)$ and $T_{d}=T_{d}(\omega)$. $T_{i}$ and $T_{d}$ are usually set proportional to $\omega^{-1}$. The problem is then reduced to the adjustment of the proportional gain $K_{p}$.

\subsection{Method 1}

The following iteration formula for the adjustment of $K_{p}$ is proposed such that the amplitude of the loop transfer function $h_{0}$ is tuned to a prescribed value $\left|h_{0}^{s}\right|$.

$$
K_{p}^{n+1}=K_{p}^{n} \frac{\left|h_{0}^{s}\right|}{\left|h_{0}^{n}\right|}=K_{p}^{n}+K_{p}^{n} \frac{\left|h_{0}^{s}\right|-\left|h_{0}^{n}\right|}{\left|h_{0}^{n}\right|}
$$

where $n$ is the index of iteration. The formula, Eqn. (1), has a quadratic convergence rate near the solution. This can be seen by using the Newton method for solving

$$
\bar{f}\left(K_{p}\right)=\left|h_{0}^{s}\right|-\left|h_{0}\right|
$$

to zero, which results in the following iteration scheme,

$$
K_{p}^{n+1}=K_{p}^{n}-\left(\frac{\partial \bar{f}}{\partial K_{p}}\right)_{n}^{-1} \bar{f}_{n}
$$

where the gradient of $\bar{f}$ with respect to $K_{p}$ is given by

$$
\frac{\partial \bar{f}}{\partial K_{p}}=-\frac{\partial\left|h_{0}\right|}{\partial K_{p}}=-\frac{1}{K_{p}}\left|h_{0}\right|
$$

Substituting Eqn. (4) into Eqn. (3) gives the iteration formula, Eqn. (1).

The adjustment formula, Eqn. (1), can be shown to be the same as the procedure for adjusting $K_{p}$ which is described in a complex manner in Schei (1991).

\subsection{Method 2}

A method for the adjustment of $K_{p}$ such that the amplitude of the sensitivity transfer function $N$ is tuned to a prescribed value $\left|N_{0}\right|$ can be derived as follows.

An iteration formula for tuning

$$
f\left(K_{p}\right)=\left|N_{0}\right|-|N|
$$

to zero, is

$$
K_{p}^{n+1}=K_{p}^{n}+\left(\frac{\partial|N|}{\partial K_{p}}\right)_{n}^{-1} f_{n}
$$

The gradient of the amplitude of $N$ with respect to $K_{p}$ can be shown to be

$$
\frac{\partial|N|}{\partial K_{p}}=-\frac{1}{K_{p}}|N|(1-\operatorname{Re}(N))
$$

See the Appendix for a proof. Substituting Eqn. (7) into Eqn. (6) gives the iteration formula

$$
K_{p}^{n+1}=K_{p}^{n}-K_{p}^{n} \frac{\left|N_{0}\right|-\left|N^{n}\right|}{\left|N^{n}\right|\left(1-\operatorname{Re}\left(N^{n}\right)\right)}
$$


A problem with this formula is that the gradient (7) may be zero in the frequency range considered. This difficulty is avoided by the following approximation.

$$
K_{p}^{n+1}=K_{p}^{n} \frac{\left|N_{0}\right|}{\left|N^{n}\right|}=K_{p}^{n}+K_{p}^{n} \frac{\left|N_{0}\right|-\left|N^{n}\right|}{\left|N^{n}\right|}
$$

This iteration scheme is found to be reasonable during simulations, and will hereafter be referred to as Method 2. Eqn. (9) is found from Eqn. (8) with $\operatorname{Re}(N)=2$. Other approximations, with different convergence properties, may be proposed by fixing $\operatorname{Re}(N)$ to a constant near the singular point.

\subsection{Method 3}

A method for the adjustment of $K_{p}$ such that the amplitude of the complementary sensitivity transfer function, $M$, is tuned to a prescribed value $\left|M_{0}\right|$ can be derived from the same procedure as Method 2. We have the iteration formula

$$
K_{p}^{n+1}=K_{p}^{n}+K_{p}^{n} \frac{\left|M_{0}\right|-\left|M^{n}\right|}{\left|M^{n}\right|\left(1-\operatorname{Re}\left(M^{n}\right)\right)}
$$

where we have used the fact that the gradient of the amplitude of $M$ with respect to $K_{p}$ can be shown to be

$$
\frac{\partial|M|}{\partial K_{p}}=\frac{1}{K_{p}}|M|(1-\operatorname{Re}(M))
$$

and that the iteration formula, Eqn. (10), is a Newton method for tuning

$$
\tilde{f}\left(K_{p}\right)=\left|M_{0}\right|-|M|
$$

to zero. See the Appendix for a proof of Eqn. (11).

If the phase angle $\angle M(j \omega)$ is prescribed, $|M|$ and $\omega$ estimated, then the iteration formula, Eqn. (10), for adjusting $K_{p}$ may be justified. Note that $\operatorname{Re}(M) \approx 1$ only for $\omega \approx 0$ which means that the iteration formula, Eqn. (10), will have no singularities in the main frequency range.

\section{Remark 1}

All proposed methods for the adjustment of $K_{p}$ may be modified to

$$
K_{p}^{n+1}=K_{p}^{n}-\lambda_{n}\left(\frac{\partial \hat{f}}{\partial K_{p}}\right)_{n}^{-1} \hat{f}_{n}
$$

where $\hat{f}$ is the performance function to be tuned to zero. The rate of convergence of the proposed methods may be improved by choosing a suitable parameter $\lambda_{n}$.

\section{Remark 2}

Assume a PID controller with $T_{i}=T_{i}(\omega)$ and $T_{d}=T_{d}(\omega)$ and that $\angle N\left(\omega, K_{p}\right)$ and $\left|N\left(\omega, K_{p}\right)\right|$ are estimated or determined from a model, $\omega$ and $K_{p}$ may then be determined directly by tuning the following set of equations to zero

$$
\begin{aligned}
& f_{1}\left(\omega, K_{p}\right)=\angle N_{0}-\angle N\left(\omega, K_{p}\right) \\
& f_{2}\left(\omega, K_{p}\right)=\left|N_{0}\right|-\left|N\left(\omega, K_{p}\right)\right|
\end{aligned}
$$


where $\angle N_{0}$ and $\left|N_{0}\right|$ are the prescribed phase and amplitude of the sensitivity transfer function, respectively. A Newton method of the same form as Eqn. (13) may be used, i.e.

$$
\theta_{n+1}=\theta_{n}-\Lambda_{n}\left(\frac{\partial f}{\partial \theta}\right)_{n}^{-1} f_{n}
$$

where $\theta=\left[\omega K_{p}\right]^{T}$ and $\boldsymbol{f}=\left[f_{1} f_{2}\right]^{T}$. The matrix $\Lambda_{n}$ is introduced to improve the convergence. The Jacobian matrix is given by

$$
\frac{\partial \boldsymbol{f}}{\partial \boldsymbol{\theta}}=\left[\begin{array}{ll}
\frac{\partial f_{1}}{\partial \omega} & \frac{\partial f_{1}}{\partial K_{p}} \\
\frac{\partial f_{2}}{\partial \omega} & \frac{\partial f_{2}}{\partial K_{p}}
\end{array}\right]=-\left[\begin{array}{ll}
\frac{\partial \angle N}{\partial \omega} & \frac{\partial \angle N}{\partial K_{p}} \\
\frac{\partial|N|}{\partial \omega} & \frac{\partial|N|}{\partial K_{p}}
\end{array}\right]
$$

This iteration scheme is based on the total differential of $\angle N$ and $|N|$, i.e.

$$
\begin{gathered}
d \angle N=\frac{\partial \angle N}{\partial \omega} d \omega+\frac{\partial \angle N}{\partial K_{p}} d K_{p} \\
d|N|=\frac{\partial|N|}{\partial \omega} d \omega+\frac{\partial|N|}{\partial K_{p}} d K_{p}
\end{gathered}
$$

The partial derivatives of $\angle N$ and $|N|$ with respect to $K_{p}$ may be determined analytically. $\partial|N| / \partial K_{p}$ is given by Eqn. (7). It can further be shown that

$$
\frac{\partial \angle N}{\partial K_{p}}=\frac{1}{K_{p}}|N| \sin (\angle N)
$$

A proof of Eqn. (20) is given in the Appendix. The partial derivatives of $\angle N$ and $|N|$ with respect to $\omega$ may be adjusted numerically during the iterations, or determined analytically if the process model is known.

\section{Examples}

\subsection{Example 1}

A PI controller is tuned for the process model

$$
h_{u}=\frac{2}{(1+s)(1+4 s)}
$$

Assume that $|N|$ and $\omega$ are estimated, the phase angle $\angle N(j \omega)$ prescribed, and that the following parameters are specified.

$$
\left|N_{\mathrm{o}}\right|=1 \cdot 5, \quad \angle N_{0}=30^{\circ}, \quad T_{i}=\frac{4}{\omega}
$$

Tuning results for Methods 2 and 3 are shown in Fig. 1. The final results are $K_{p}=1 \cdot 94$, $T_{i}=3.4$ and $\omega=1 \cdot 18$. For this example Method 1 did not converge.

For this example, Fig. (1) shows that Methods 2 and 3 have approximately equal rates of convergence. Now, assume that $|M|$ and $\omega$ are estimated, the above specifications are then identical to

$$
\left|M_{0}\right|=0 \cdot 81, \quad \angle M_{0}=-111 \cdot 7^{\circ}, \quad T_{i}=\frac{4}{\omega}
$$



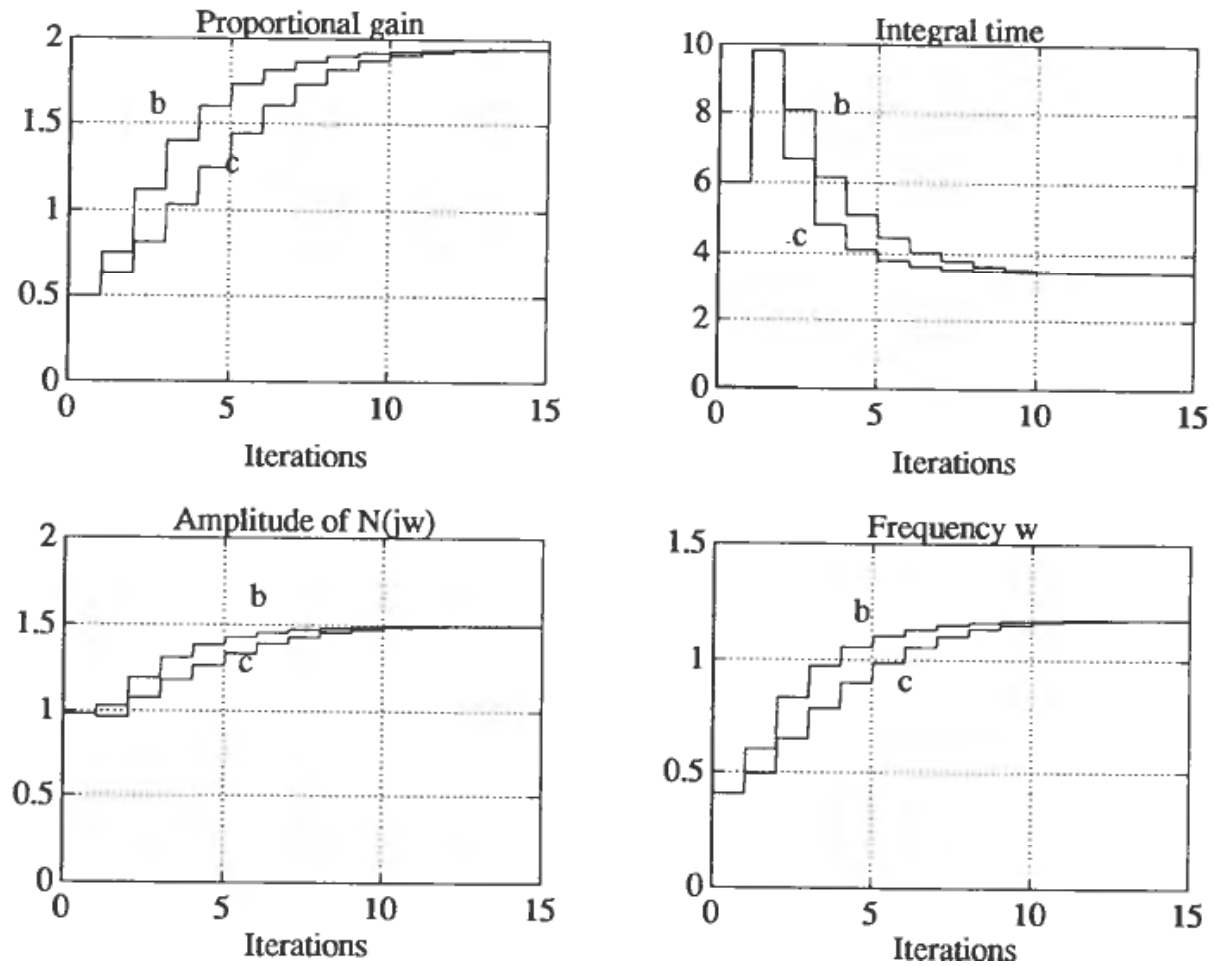

Figure 1. $K_{p}, T_{i},|N|$ and $\omega$ during tuning of the PI controller, for b, Method 2 and c, Method 3.
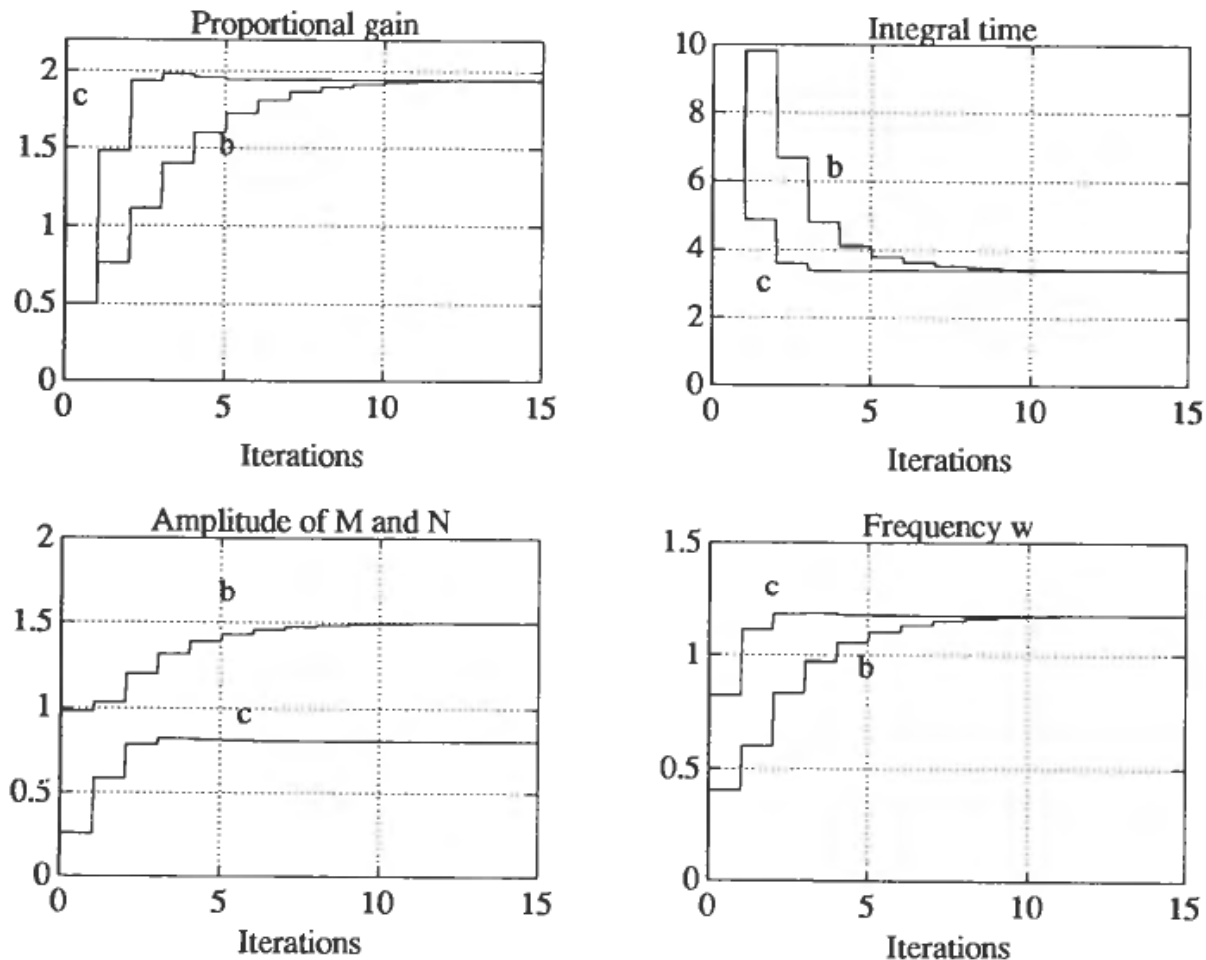

Figure 2. $K_{p}, T_{i},|N|,|M|$ and $\omega$ during tuning of the PI controller, for b, Method 2 and c, Method 3. 
Simulation results based on Method 3 for estimation of $|M|$ and Method 2 for estimation of $|N|$ are shown in Fig. 2.

Figure 2 shows that Method 3 based on esimation of $|M|$ has a considerably faster rate of convergence compared to Method 2, based on estimation of $|N|$. However, the convergence may be improved by the use of the modified scheme, Eqn. (13), with a suitable parameter $\lambda_{n}$.

\subsection{Example 2}

A PI controller is tuned for the process model

$$
h_{u}=\frac{2}{(1+s)(1+4 s)} e^{-s}
$$

Assume that $|N|$ and $\omega$ are estimated, the phase angle $\angle N(j \omega)$ prescribed, and that the following parameters are specified.

$$
\left|N_{0}\right|=2, \quad \angle N_{0}=0^{\circ}, \quad T_{i}=\frac{5}{\omega}
$$

Tuning results for Methods 1,2 and 3 are shown in Fig. 3. All methods converged for this example. The final results are $K_{p}=1.24, T_{i}=5.52$ and $\omega=0.91$. Note that the rate of convergence is considerably faster than for Example 1. This example indicates faster convergence for processes with a time delay, because the frequency, $\omega$, is nearly constant during the adaptation of the controller parameters. Compare this to Example 1.
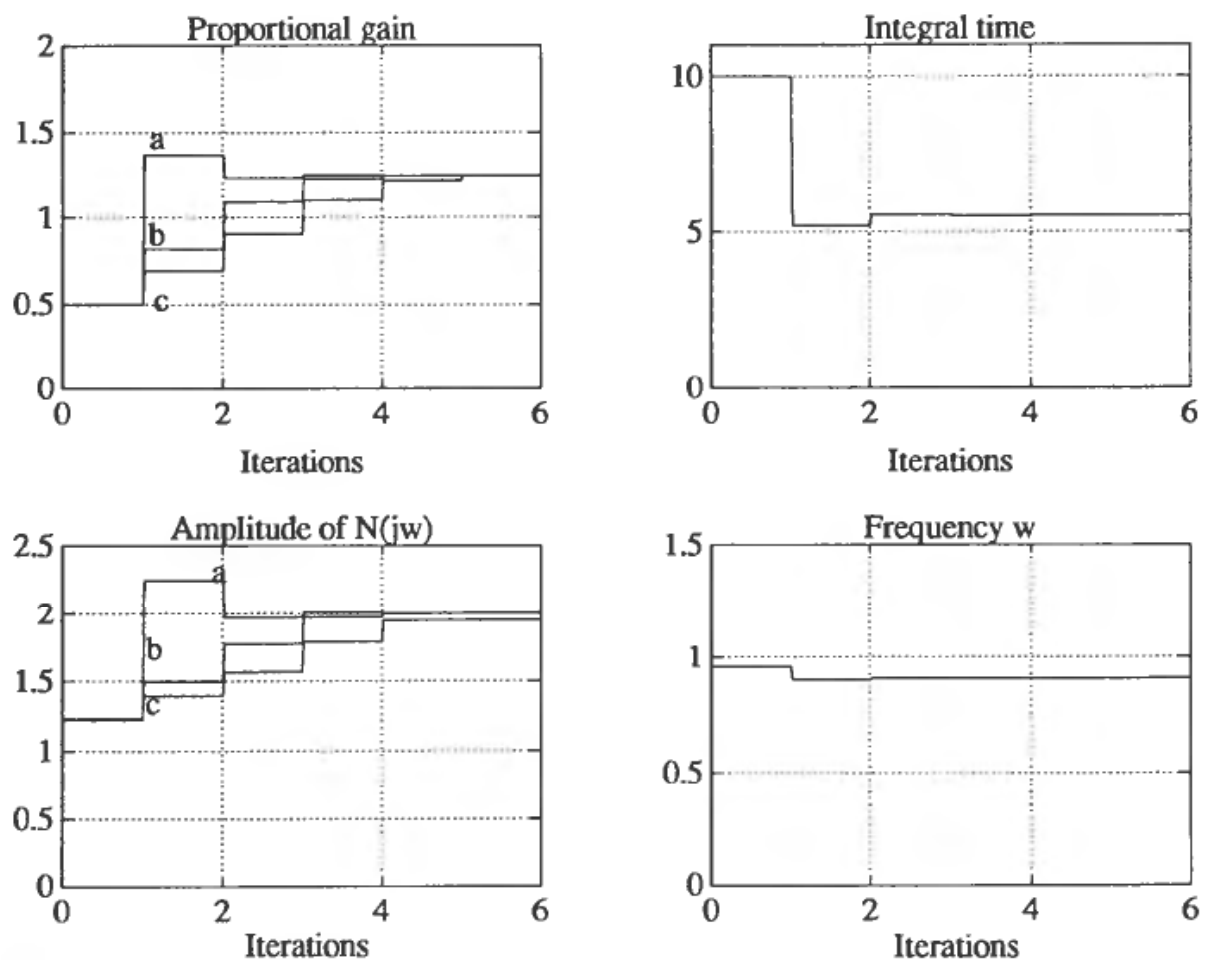

Figure 3. Figure 3. $K_{p}, T_{i},|N|$ and $\omega$ during tuning of the PI controller, for a, Method 1; b, Method 2 and c, Method 3. 


\section{Concluding remarks}

Three methods based on gradient informations for the adjustment of the proportional gain in a PID controller are presented. The integral time constant, and the derivative time constant, are set proportional to the inverse of the frequency.

A method for the determination of PID parameters presented in a complicated way in Schei (1991) is shown to be a simple Newton method for the adjustment of $K_{p}$ such that the amplitude of the loop transfer function $h_{0}(j \omega)$ is tuned to a specified value.

Simple analytical expressions for the gradient of the amplitude of the sensitivity transfer function $N(j \omega)$ and the complementary sensitivity transfer function $M(j \omega)$ with respect to the proportional gain $K_{p}$ are presented. These expressions are the basis for two Newton methods for the adjustment of the proportional gain.

It is shown that the proportional gain should be adjusted from gradient information of $|M|$ rather than gradient information of $|N|$. This statement is justified from the fact that the gradient of $|N|$ with respect to $K_{p}$ may be zero in the main frequency range, which means that the Newton method will be singular. However, an approximate iteration formula based on $|N|$, which may be used, is presented.

\section{REFERENCES}

Åstrøm, K. J., and HäGglund, T. (1984). Automatic tuning of simple regulators with specifications on phase and amplitude margins. Automatica, 20, 645-651.

Balchen, J. G., Telnes, K., and Di Ruscio, D. (1989). Frequency response adaptive control of a refrigeration cycle. Modeling, Identification and Control, 10, 3-11.

SCHEI, T. S. (1991). A new method for automatic tuning of PID control parameters. Proceedings of the 1st ECC-European Control Conference, Grenoble, 2-5 July 1991, pp. 1522-1527.

\section{Appendix}

A proof of Eqn. (7)

The sensitivity transfer function is given by

$$
N(j \omega)=\frac{1}{1+h_{0}(j \omega)}
$$

Define $h=\left|h_{0}(j \omega)\right|, \phi=\angle h_{0}(j \omega)$ and $h_{0}(j \omega)=h e^{j \phi}$ to simplify the notation. We have

$$
N(j \omega)=\frac{1}{1+h e^{j \phi}}=\frac{1+h e^{-j \phi}}{\left(1+h e^{j \phi}\right)\left(1+h e^{-j \phi}\right)}=\frac{1+h e^{-j \phi}}{1+h\left(e^{j \phi}+e^{-j \phi}\right)+h^{2}}
$$

which may be written

$$
N(j \omega)=\frac{1+h \cos (-\phi)+j h \sin (-\phi)}{1+h^{2}+2 h \cos (\phi)}=\operatorname{Re}(N)+j \mathscr{I} m(N)
$$

The amplitude of $N(j \omega)$ is given by

$$
|N|=\frac{\left[(1+h \cos (-\phi))^{2}+(h \sin (-\phi))^{2}\right]^{1 / 2}}{1+h^{2}+2 h \cos (\phi)}
$$

or

$$
|N|=\frac{1}{\sqrt{\left(1+h^{2}+2 h \cos (\phi)\right)}}
$$


The gradient of $|N(j \omega)|$ with respect to $K_{p}$ is

$$
\frac{\partial|N|}{\partial K_{p}}=-\frac{1}{2} \frac{1}{\left(1+h^{2}+2 h \cos (\phi)\right)^{3 / 2}}\left[(2 h+2 \cos (\phi)) \frac{\partial h}{\partial K_{p}}-2 h \sin (\phi) \frac{\partial \phi}{\partial K_{p}}\right]
$$

For a PID controller we have

$$
\frac{\partial h}{\partial K_{p}}=\frac{1}{K_{p}} h
$$

and

$$
\frac{\partial \phi}{\partial K_{p}}=0
$$

when $\omega$ is constant. Substituting Eqns. (32), (33) and (30) into Eqn. (31) yields

$$
\frac{\partial|N|}{\partial K_{p}}=-\frac{1}{K_{p}}|N|\left(1-\frac{1+h \cos (\phi)}{1+h^{2}+2 h \cos (\phi)}\right)
$$

which is seen to be equal to

$$
\frac{\partial|N|}{\partial K_{p}}=-\frac{1}{K_{p}}|N|(1-|N| \cos (\angle N))
$$

by the use of Eqn. (28), and Eqn. (7) is proved.

A proof of Eqn. (11)

The complementary sensitivity transfer function is given by

$$
M(j \omega)=\frac{h_{0}(j \omega)}{1+h_{0}(j \omega)}=\frac{h^{2}+h \cos (\phi)+j h \sin (\phi)}{1+h^{2}+2 h \cos (\phi)}=\operatorname{Re}(M)+j \mathscr{I} m(M)
$$

The amplitude of $M(j \omega)$ is

$$
|M|=\frac{h}{\sqrt{ }\left(1+h^{2}+2 h \cos (\phi)\right)}
$$

The gradient of $|M(j \omega)|$ with respect to $K_{p}$ is

$$
\frac{\partial|M|}{\partial K_{p}}=\frac{\frac{\partial h}{\partial K_{p}}\left(1+h^{2}+2 h \cos (\phi)\right)-h\left[(h+\cos (\phi)) \frac{\partial h}{\partial K_{p}}-h \sin (\phi) \frac{\partial \phi}{\partial K_{p}}\right]}{\left(1+h^{2}+2 h \cos (\phi)\right)^{3 / 2}}
$$

which, by the use of Eqns. (32) and (33), may be shown to be

$$
\frac{\partial|M|}{\partial K_{p}}=\frac{1}{K_{p}}|M|\left(1-\frac{h^{2}+h \cos (\phi)}{1+h^{2}+2 h \cos (\phi)}\right)
$$

or

$$
\frac{\partial|M|}{\partial K_{p}}=\frac{1}{K_{p}}|M|(1-|M| \cos (\angle M))
$$

and Eqn. (11) is proved.

Note that, $\operatorname{Re}(N)+\operatorname{Re}(M)=1$, which may be substituted into Eqns. (7) and (11) for the derivation of alternative expressions. 
A proof of Eqn. (20)

From Eqn. (28) we have

$$
\tan (\angle N)=\frac{h \sin (-\phi)}{1+h \cos (-\phi)}
$$

The partial derivative of the right-hand side of Eqn. (41) with respect to $K_{p}$ is given by

$$
\frac{\partial \tan (\angle N)}{\partial K_{p}}=\frac{1}{K_{p}}|N| \frac{\tan (\angle N)}{\cos (\angle N)}
$$

The chain rule on the left-hand side of Eqn. (41) gives

$$
\frac{\partial \tan (\angle N)}{\partial K_{p}}=\frac{1}{\cos ^{2}(\angle N)} \frac{\partial \angle N}{\partial K_{p}}
$$

Combining Eqns. (42) and (43) gives

$$
\frac{\partial \angle N}{\partial K_{p}}=\frac{1}{K_{p}}|N| \sin (\angle N)
$$

and Eqn. (20) is proved. 